\title{
Neuropatía Periférica en la enfermedad de Parkinson
}

\author{
Peripheral Neuropathy in Parkinson's disease
}

\author{
Marcela Alvarado 1,a
}

\section{RESUMEN}

La enfermedad de Parkinson es una entidad neurodegenerativa con alteraciones principalmente extrapiramidales, del sistema nervioso autónomo y, en etapas finales, alteraciones cognitivas y neuropsiquiátricas. Desde 1976 se reconocen además síntomas neurosensoriales dependientes del sistema nervioso periférico, presentes en casi la mitad de los pacientes y que preceden incluso a los síntomas motores. Los síntomas sensitivos fueron inicialmente atribuidos a distonías o a niveles indebidos de levodopa en ausencia de medicación apropiada; sin embargo, estudios recientes sugieren una etiología intrínseca a la propia enfermedad. Se reconocen tres formas de presentación: polineuropatía crónica (la más común), aguda y subaguda. La clínica de la polineuropatía es concurrente con la de la enfermedad de Parkinson y el diagnóstico es básicamente clínico, aun cuando se cuenta con el apoyo de la electromiografía y de la velocidad de conducción nerviosa, cuya normalidad no descartaría a esta entidad. El manejo actual está basado en la presunción etiológica, por lo que la reposición de vitaminas como la B12, B1 y folatos puede considerarse como parte del mismo. La etiología, el pronóstico y la fisiopatología son aún temas de debate y requieren, por lo tanto, más investigaciones.

PALABRAS CLAVE: Polineuropatías, enfermedad de Parkinson, neuropatía periférica, sistema nervioso periférico.

\section{SUMMARY}

Parkinson's disease is a neurodegenerative entity with predominant extrapyramidal and dysautonomic features and, in the final stages, cognitive and neuropsychiatric manifestations. Since 1976, the involvement of the peripheric nervous system has been recognized, mainly through neurosensorial symptoms affecting almost half of the Parkinson patients, even before the onset of motor symptoms. Initially, these symptoms were attributed to dystonia or the use of levodopa in the absence od medications; however, recent studies suggest an intrinsic etiology in idiopathic Parkinson's disease. Chronic (the most frequently described), subacute and acute polyneuropathy are the main clinical forms. The sensory symptoms coexist with those of Parkinson's disease. The diagnosis is essentially based on a clinical examination supported by electromyography and tests of neuro-conduction velocity whose normality does not rule out this entity, however. Its management depends on the etiological assumptions, and includes the eventual use of vitamin B12, B1 and folates replacement therapy. The etiology, the prognosis and the physiopathology are still topics of debate whose clarification requires further research.

KEYWORDS: Polyneuropathies, Parkinson disease, peripheral neuropathy, peripheral nervous system.

\footnotetext{
Hospital de Emergencias de Villa el Salvador. Lima, Perú.

a Médica neuróloga
} 


\section{INTRODUCCIÓN}

La Enfermedad de Parkinson (EP) fue descrita en 1817 por James Parkinson, es una enfermedad neurodegenerativa caracterizada por alteraciones a nivel extrapiramidal, principalmente temblor de reposo, bradicinesia, rigidez, inestabilidad postural. Otras alteraciones relacionadas con disfunción del sistema nervioso autónomo, incluyen sudoración, sialorrea y ulteriormente alteraciones cognitivas que pueden conllevar a la demencia en etapas finales de la enfermedad. La enfermedad de Parkinson asocia tambien calambres musculares y entumecimiento, siendo más prominentes en region distal de los miembros. Estos síntomas, son características de las neuropatías distales, principalmente las sensoriales. Esta superposición entre las características clínicas de ambas entidades han dificultado la identificación de la concurrencia de la afectación del sistema nervioso periférico como una causa adicional que afecta la calidad de vida en pacientes con enfermedad de Parkinson (1).

Las manifestaciones sensoriales en la enfermedad de Parkinson están descritas desde 1976, semejando aquellas que se presentan en la neuropatía periférica. Se ha descrito que casi la mitad de los pacientes con enfermedad de Parkinson idiopática presentan estos síntomas. Estas manifestaciones sensoriales, principalmente el dolor, habrían sido atribuídas a otras causas como a la coexistencia de distonías focales, o bien lo relacionaban a la discontinuación o reducción de la medicación (2).

Estudios recientes sugieren la exisencia de compromiso del sistema nervioso periférico en EP, por lo que se pueden asociar neuropatías, aunque este riesgo debe ser individualizado para cada paciente. Se ha descrito una mayor ocurrencia de neuropatía en pacientes con enfermedad de Parkinson comparados con controles sanos, mayormente asociada a la deficiencia de vitamina B12 causada por la exposición a levodopa. La discriminación etiológica entre la exposición acumulada a levodopa y la severidad de la EPes aun un desafio. Algunos pacientes con enfermedad de Parkinson recién diagnosticada (sin antecedentes de exposición a fármacos antiparkinsonianos) tienen, sin embargo, una mayor ocurrencia de neuropatía que la observada en controles de población general. Se ha sugerido que las deficiencias de vitaminas B6, B12 y / o folato podrían jugar un papel importante en la neuropatía asociada a la enfermedad de Parkinson; por otro lado, otros autores defienden la hipótesis etiológica de que la causa de la neuropatía sea intrínseca a la EP en si misma (3).

Se ha sugerido que la rigidez y el temblor, de reposo podrían ser desencadenantes de algunas lesiones en nervio periférico debido a la intensidad y magnitud de los mismos y por ocasionar traumas repetidos. La mano estriatal y las deformidades del pie, secundarias a distonía y rigidez parkinsoniana, estarían asociados a mayor severidad de la EP y serían responsables de causar discapacidad funcional por lesiones en nervio mediano y ulnar. El temblor y la rigidez parkinsoniana afectarían la transmisión neuronal contribuyendo a la progresión de la neuropatía (4).

El objetivo de esta revisión es describir la evidencia disponible sobre el compromiso de los nervios periféricos en pacientes con enfermedad de Parkinson.

\section{Neuropatía en enfermedad de Parkinson en la actualidad:}

En la última década ha surgido una atención cada vez mayor en relación a la presencia de neuropatía en pacientes con enfermedad de Parkinson, debido a que la concurrencia de ambas entidades es bastante común. Existe controversia de si es parte del proceso neurodegenerativo en sí, es decir intrínseca de la EP o si es parte de una complicación secundaria al uso de levodopa entre otros factores asociados $(5,6)$.

El uso de levodopa vía oral se ha asociado con neuropatía principalmente con polineuropatía crónica, aunque más raramente con formas agudas o subagudas. La administración de levodopa en infusión en gel intestinal, alternativa terapéutica para mejorar la administración de levodopa, se ha asociado con más casos de neuropatía periférica(7).

\section{Epidemiología}

La prevalencia de neuropatía periférica en la población general es del 2,4\%, pudiéndose elevar hasta un $8 \%$ en poblaciones de mayor edad, con diabetes mellitus, y la causa más común como son las formas idiopáticas (2). La prevalencia de enfermedad de Parkinson es de 0,1-0,2\% de la población general, elevándose al $1 \%$ en los mayores de 60 años de edad (2). Por consiguiente, solamente un pequeño porcentaje de la población general puede tener concurrencia de parkinsonismo y polineuropatía que se estima menor a $0,01 \%$ de los mayores de 60 años basándose solo en el azar; pero si consideramos la prevalencia puntual 
de neuropatía del total de pacientes con diagnóstico de EP se informa que es tan alto como el 55\% (rango $37,8 \%-55 \%$ ) es por esto su gran importancia e interés científico $(8,9)$.

Inicialmente la neuropatía en la enfermedad de Parkinson se consideraba como de escasa relevancia epidemiológica, y estaba relegada a los limitados y raros casos de Parkinson genético por variantes patogénicas en el gen $P R K N$, enfermedades mitocondriales con parkinsonismo o familias con síndrome de parkinsonismo con neuropatía. Actualmente existen evidencia creciente de compromiso de nervio periférica en EP (6).

Un estudio Ingles publicado en el 2011 comparó 37 pacientes con EP de los cuales 14 (37,8\%) tenían neuropatía, con 37 pacientes controles de los cuales $3(8,1 \%)$ presentaban neuropatía $(\mathrm{p}<0.005)$. En este estudio encontraron que la medición de vitamina B12 era baja en ambos grupos de estudio, pero que la medición fue significativamente menor en el grupo con polineuropatía y enfermedad de Parkinson respecto de los controles que tenían polineuropatía asociada a otras entidades no parkinsonianas, sugiriendo una relación con la exposición acumulativa de levodopa (10).

Posteriormente, en el 2013 la " Parkinson Disease and other Movement Disorders Society o MDS" publicó un estudio multicéntrico en Italia, evaluando 330 pacientes con enfermedad de Parkinson y 137 controles, encontró la ocurrencia de polineuropatía en $19,40 \%$ de los pacientes con exposición prolongada a levodopa (3 a más años), 6,80\% de los pacientes con exposición corta a levodopa (menor a 3 años), 4,82\% de los pacientes sin exposición a levodopa y $8,76 \%$ de los del grupo control. En este estudio se encontró la neuropatía axonal predominantemente sensorial como el tipo mas frecuente. Asimismo, se encontró un aumento del riesgo de neuropatía en aproximadamente un $8 \%$ por cada año de edad (1). Asimismo, se sugiere la frecuencia de neuropatía se incrementa con una mayor exposición a levodopa, con niveles bajos de vitamina B12, y con niveles altos de homocisteína (6). Asimismo, en el Reino Unido (2015-2018) encontraron una frecuencia 2.4 veces mayor de polineuropatia en pacientes con EP (15 de 5089, 0.29\%) respecto de controles (24 de 19 897, 0.12\%), en este estudio proponen que la presencia de polineuropatía no estaba relacionada a los efectos adversos de fármacos antiparkinsonianos (3).

Se han reportado casos muy raros y anecdóticos de polineuropatía aguda y subaguda en pacientes con $\mathrm{EP}$, informado en pacientes que recibieron infusión intraduodenal continua de levodopa/carbidopa en gel intestinal, y aparentemente no relacionados a infecciones previas; algunos de los casos reportados aparentemente asociados a causas nutricional secundarias al uso de la infusión intestinal de la levodopa (11).

\section{Fisiopatología}

Se considera que la deficiencia de vitaminas B6, B12 y/o folatos podría jugar un rol importante en la neuropatía asociada a la EP (3). El sello

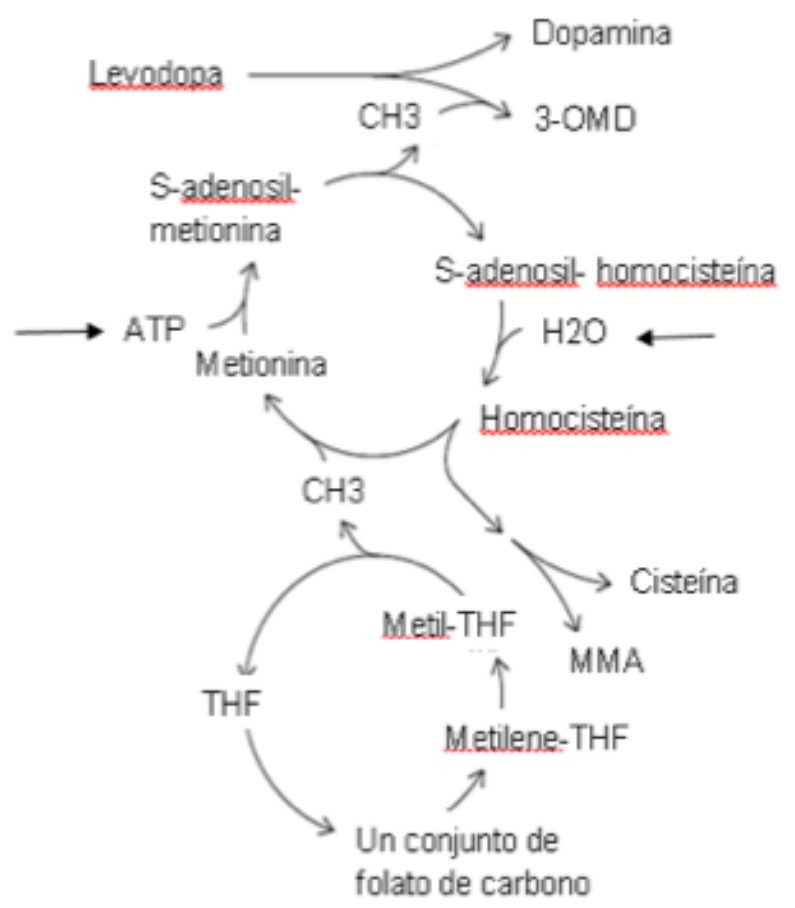

Conversión de levodopa en 3-O-metildopa (3-OMD) agota las reservas de grupos metilo $(\mathrm{CH} 3)$ y conduce a producción de homocisteína. Re-metilación de homocisteína posterior (en metionina) requiere vitamina B12 (cobalamina) como cofactor y obtiene su $\mathrm{CH} 3$ del conjunto de folato de carbono. Implicación del metilentetrahidrofolato (metileno-THF) en el suministro del $\mathrm{CH} 3$ hace que el polimorfismo en la reductasa de metileno-THF sea importante determinante del nivel de homocisteína en plasma. Transulfuración de homocisteína (en cisteína) requiere vitamina B6 (piridoxina). Una ruta que conduce al ácido metilmalónico(MMA) hace que tanto la MMA como la homocisteína sean marcadores del déficit funcional de la vitamina B12. ATP = trifosfato de adenosina.

Figura 1. Facetas del metabolismo de levodopa y homocisteína en la neuropatía periférica en pacientes con enfermedad de Parkinson (11). 
histológico de la EP es la presencia de inclusiones intracitoplasmáticas conocidas como cuerpos de Lewy, compuestos principalmente por agregados de alfa sinucleína, cuya función no es completamente conocida pero se cree que entre otras cosas produce una acción pro inflamatoria en las células microgliales, y que además los depósitos de alfa sinucleína puede afectar el transporte axonal; dado que el sistema nervioso periférico es un objetivo de los depósitos de alfa sinucleína, existe un interés creciente en evaluar si son las características intrínsecas de la EP lo que puede predisponer a neuropatías periféricas o está en relación a factores asociados como la exposición a levodopa o el déficit de vitamina B12, o ambos (12).

Existe evidencia de una fuerte asociación entre neuropatía y el aumento de los niveles de homocisteína. El aumento de los niveles de homocisteína durante el uso de levodopa oral o en infusión duodenal está relacionado con el consumo de grupos metilo por COMT. Tanto la vitamina B12, B6 y folatos son cofactores necesarios para el metabolismo de la homocisteína. La deficiencia de estos cofactores estaría relacionado con efectos tóxicos en los nervios periféricos al aumentar la vulnerabilidad a las toxinas mitocondriales y de radicales libres induciendo reacciones inflamatorias y alterando los mecanismos de reparación del ADN $(1,6)$. La levodopa podría favorecer el daño a los nervios al interactuar con el ciclo de la homocisteína/vitamina B12. La conversión de levodopa a dopamina requiere una donación del grupo metilo, proporcionado por la adenosilmetionina, que conduce a la formación de homocisteína. Posteriormente, la remetilación de la homocisteína requiere como cofactor a la vitamina $\mathrm{B} 12$, y formas alternativas de degradación requieren a la metilentetrahidrofolato y a la piridoxina, por lo tanto el uso crónico de levodopa conlleva a la depleción del grupo metilo y acumulación de homocisteína (12) (figura 1).

En pacientes con EP se ha descrito una rápida acumulación de alfa sinucleína a nivel del nervio motor faríngeo y en la rama sensorial del vago, asi como en nervio glosofaríngeo y en el nervio laríngeo superior de pacientes con disfagia $(5,12)$. Se ha demostrado que en los pacientes con EP existe una pérdida significativa de fibras nerviosas epidérmicas y corpúsculos de Meissner, en comparación con controles sanos (3). A nivel de las fibras cutáneas se detectaron depósitos de alfa sinucleína, lo cual se correlaciona con disfunción sensorial, además existen agregados de alfa sinucleína en fibras amielínicas de la dermis, también en el aparato pilomotor y sudomotor (12).
Recientemente se consideró una variación en cuanto a la teoría de Braak, en el cual se propone que el inicio de la EP no estaría en el tronco cerebral sino en el sistema nervioso periférico, en particular a nivel del nervio olfatorio y especialmente en el entérico, ya que precisamente en estas áreas existe tempranamente acúmulo de alfa sinucleína. Asimismo, se propone que la neurodegeneración empezaría cuando un patógeno extraño ingresa al organismo ya sea por la ruta nasal o la gástrica, e induzca la alteración de la alfa sinucleína tomando una conformación alterada y propagándose a las neuronas vulnerables inicialmente cercanas y luego al sistema nervioso central por medio de las fibras vagales preganglionares por un mecanismo retrógado. Esta explicación ha tomado mucho interés por la comunidad científica, ya que es respaldada por varios estudios, entre los más interesantes tenemos un estudio danés en el que un patógeno neurotrópico penetra en el sistema nervioso central por medio del sistema nervioso periférico a través de su ascenso por el nervio vago y que es por ello que la resección completa del vago está asociada con disminución del riesgo de desarrollar enfermedad de Parkinson (6).

Algunos estudios proponen que la pérdida de fibras nerviosas periféricas es una característica intrínseca de la enfermedad de Parkinson. En estudios neuropatológicos se determinó dos tipos de neurodegeneración periférica en la enfermedad de Parkinson: a) Reducción de fibras nerviosas pequeñas intraepidermicas dependientes de longitud y b) Severa reducción de fibras nerviosas intraepidermicas no dependientes de longitud, coindicdente con las manifestaciones proximales más pronunciadas.

Histopatológicamente los depósitos de alfa sinucleína se han encontrado tanto a nivel del sistema nervioso periférico como en las fibras nerviosas dérmicas, intraepidérmicas, glándulas sudoríparas, músculos pilo erectores, fibras somatosensoriales, nervios faríngeos, nervios de las glándulas, nervios salivales submandibulares y ganglios del sistema nervioso entérico; llama la atención que nunca se observó depósitos de alfa sinucleína en fibras mielinizadas (5).

Además se ha descrito la asociación de neuropatía periférica con la EP genético de inicio temprano asociada a variantes patogénicas del gen $P R K N$, sugiriendo un vínculo entre la degeneración neuronal central y la periférica, por lo menos en estos subgrupos de pacientes con enfermedad de Parkinson $(1,3)$. En éste grupo genéticamente predispuesto se puede 
desarrollar una deficiencia de vitamina B12 inducida por levodopa secundaria a la acumulación de los niveles de ácido metilmalónico (10).

En pacientes con enfermedad de Parkinson con la variante p.A53T-SNCA se presentan neuronas con agregados proteicos, alteración en la formación de las neuritas, axones contorsionados o fragmentados, hinchados con varicosidades que contienen alfa sinucleína y proteínas Tau. Estas neuronas presentan conectividad sináptica disminuida, con axones distróficos y alteración en el transporte axonal (13).

Existe evidencia de que la neuropatía en la EP estaría asociada con un grupo de citopatías mitocondriales, relacionadas con variantes genéticas específicas como la "epilepsia mioclónica de fibras rojas rasgadas" que presentan parkinsonismo con respuesta a levodopa, pérdida de audición, neuropatía, miopatía y biopsia con fibras rojas rasgadas, o la ataxias espinocerebelosa tipo 3 cuyo fenotipo parkinsoniano que responde a levodopa, polineuropatía sensorial-motora y oftalmoplejía progresiva (14).

Los mecanismos propuestos para explicar la polineuropatía relacionada con la infusión en gel intestinal de levodopa/carbidopa, incluyen la obstaculizacíon de la membrana yeyunal promoviendo la malabsorción de vitaminas, incremento de niveles de homocisteina neurotóxica, asi como la asociación entre la gastrostomía percutánea y activación de mecanismos disinmunes(7).

La polineuropatía inflamatoria aguda en EP es aun más controvertida, una posible explicación sería que la deficiencia de vitamina B12 y el aumento de la homocisteína determinan un desequilibrio agudo entre factores mielinotóxicos y tróficos, con posterior aumento de citoquinas proinflamatorias, toxinas, radicales libres y disminución de la síntesis de factores de crecimiento (9).

\section{Características clínicas}

La definición de las características clínicas en esta entidad es compleja debido a la amplia superposición de los síntomas de un cuadro de neuropatía con las características no motoras presentes en un paciente con EP. Los sintomas incluyen parestesias simétricas y distales, síntomas sensoriales al inicio de la enfermedad, alteración de la percepción del dolor y de la temperatura, reflejos tendinosos preservados (al inicio de la enfermedad) y alteración del equilibrio
$(1,15)$. Estos síntomas tienden a ser progresivos, con afectación ulterior de las fibras nerviosas grandes que conducen a la pérdida de los reflejos y debilidad muscular a nivel de pies y piernas, y en casos más severos las manos y el torso. Habitualmente se le considera como neuropatía axonal pero también podría afectar a la mielina y en algunos casos a ambos dentro de las fibras nerviosas (16). La neuropatía en enfermedad de Parkinson puede incluir mononeuropatías, polineuropatías y ganglionopatías, dependientes de parámetros etiológicos (17).

La neuropatía periférica se define como simétrica, progresiva y de predominio distal y se puede presentar en todos los estadios de la EP. El riesgo de polineuropatías no se ven influenciadas por la edad, sexo, duración de la enfermedad, puntaje de UPDRS-III (11). Sin embargo, una vez adquirida la polineuropatía tanto la edad como la duración prolongada de la enfermedad pueden jugar un papel importante en la severidad del cuadro (18).

En los casos raros de polineuropatía aguda o subaguda en pacientes con enfermedad de Parkinson se presenta predominantemente alteración sensoriomotora distal ascendente de tipo tetraparesia flácida, parestesias, entumecimiento, ataxia, pero no es una presentación usual y su presencia es anecdótica (11). Los pocos casos de polineuropatía aguda relacionada a enfermedad de Parkinson no han sido relacionados al antecedente de infección, a excepción de un caso reportado, también se reportaron casos de polineuropatía aguda en pacientes que recibieron infusión en gel de levodopa/carbidopa a los 7 meses del inicio de la infusión (19) (tabla 1).

\section{Clasificación}

La neuropatía en la enfermedad de Parkinson principalmente se presenta como polineuropatía, la cual puede darse en tres presentaciones: a) Polineuropatía aguda en EP (duración de pocas horas hasta las tres primeras semanas), b) Polineuropatía subaguda (periodo $<60$ días) y c) Polineuropatía cronica en EP (periodo $>=60$ días).

Si la polineuropatía está en relación a la toma de levodopa, se puede clasificar en relación al tiempo de exposición a levodopa en: a) Polineuropatía sin exposición a levodopa b) Polineuropatía con exposición larga a levodopa: cuando el paciente ha sido expuesto a la levodopa por más de 3 años, y c) Polineuropatía con exposición corta a levodopa: 
Tabla 1. Resumen de las características principales y las implicaciones clínicas actuales y futuras para cada aspecto de la neuropatía de la EP (5).

\begin{tabular}{llll}
\hline Factor causante & $\begin{array}{l}\text { Fibras nerviosas } \\
\text { involucradas }\end{array}$ & Características clínicas & $\begin{array}{l}\text { Implicaciones clínicas actuales y } \\
\text { futuras }\end{array}$ \\
\hline Levodopa & Largas (fibras A) & $\begin{array}{l}\text { Parestesias, } \\
\text { empeoramiento del } \\
\text { equilibrio }\end{array}$ & $\begin{array}{l}\text { Utilidad del suplemento con } \\
\text { vitaminas y prevención con ICOMT }\end{array}$ \\
Intrínseca & $\begin{array}{l}\text { Pequeñas amielínicas } \\
\text { (fibras C) }\end{array}$ & $\begin{array}{l}\text { Deterioro del umbral } \\
\text { sensorial del dolor }\end{array}$ & $\begin{array}{l}\text { Utilidad de la biopsia cutánea para el } \\
\text { diagnóstico precoz de la enfermedad } \\
\text { de Parkinson }\end{array}$ \\
$\begin{array}{l}\text { Patógeno extraño / } \\
\text { Daño ambiental }\end{array}$ & $\begin{array}{l}\text { Terminaciones nerviosas } \\
\text { vagal y olfativa }\end{array}$ & Constipación e hiposmia & $\begin{array}{l}\text { Papel causal en el inicio y la difusión } \\
\text { del proceso de neurodegeneración. } \\
\text { Posible objetivo de terapias } \\
\text { innovadoras }\end{array}$ \\
\hline
\end{tabular}

cuando el paciente ha sido expuesto a levodopa por menos de 3 años. $(1,6)$

En caso de presentar un paciente sin manifestaciones clínicas y que desarrolla sólo alteraciones electrofisiológicas se clasificaría como polineuropatía en enfermedad de Parkinson de tipo subclínico (7).

\section{Diagnóstico}

El diagnóstico de neuropatia asociada a EP resulta complejo y poco preciso, debido a la gran similitud clínica entre la polineuropatía y las características no motoras en pacientes con EP. Condiciones asociadas como la edad, el déficit de vitamina B12, B6, folatos, la exposición a levodopa, entre otros nos permite sospechar en esta patología. Ante la sospecha, se debe solicitar estudios electrofisiológicos que demuestren parámetros anormales en al menos uno de los nervios explorados en correlación con la clínica (1).

Se debe evaluar la fuerza muscular, reflejos osteotendinosos, ataxia, alteración de la percepción de la sensibilidad termoalgésica, táctil, vibración y posición. Tambien podemos apoyarnos de escalas para la evaluación de polineuropatía como la del sistema de evaluación clínica de Toronto o usando los criterios de diagnóstico desarrollados por la Academia Americana de Neurología, que nos permita clasificar el tipo de polineuropatía (figura 2).

Entre los exámenes de laboratorio que deberían solicitarse tenemos: hemograma completo, glucosa en ayunas, prueba de tolerancia oral a la glucosa, electrolitos, creatinina, hormonas tiroideas, pruebas de función hepática, hepatitis $\mathrm{B}$ y $\mathrm{C}$, anticuerpos antinucleares, homocisteína sérica en ayuno, vitamina B12, B1, B6 y niveles de ácido fólico, entre otros según los consideremos relevante para descartar otras posibles causas de la polineuropatía, es decir posibles diferenciales (15).

En la mayoría de los pacientes con polineuropatía en la EP se observó una reducción de la amplitud del nervio sural, igualmente el deterioro del elemento sensorial fue mayor que del elemento motor (el cual era normal o discretamente alterado en la mayoría de pacientes) (1), al inicio la velocidad de conducción fue normal, esto puede ir cambiando durante el seguimiento del paciente. La neuropatía axonal es el hallazgo más común, cabe resaltar que si comparamos a pacientes que usan levodopa vía oral vs los que la usan en infusión en gel de levodopa/carbidopa los hallazgos fueron más severos en pacientes tratados con infusión de gel de levodopa/carbidopa. En este grupo de pacientes el cambio neuropático se correlacionó con pérdida de peso desde el inicio del tratamiento y con la dosis del fármaco (16), por lo que se recomienda vigilancia de los cambios en el índice de masa corporal.

Durante el seguimiento clínico podemos apoyarnos de la escala UPDRS-III para valorar impacto funcional. El empeoramiento durante el seguimiento lo podemos valorar con la neurografía la cual se define como una significativa reducción de la amplitud de los potenciales de acción de estudios de conducción nerviosa $(>=50 \%)$ y/o velocidades de conducción nerviosa $(>=20 \%)$ en al menos dos nervios motores y/o sensitivos (7).

Los criterios para neuropatía en enfermedad de Parkinson son menos rígidos y pueden ser diagnosticados únicamente por la clínica del paciente (13). Un hallazgo electriofisiológico negativo en un paciente con alta sospecha clínica, no descarta esta 


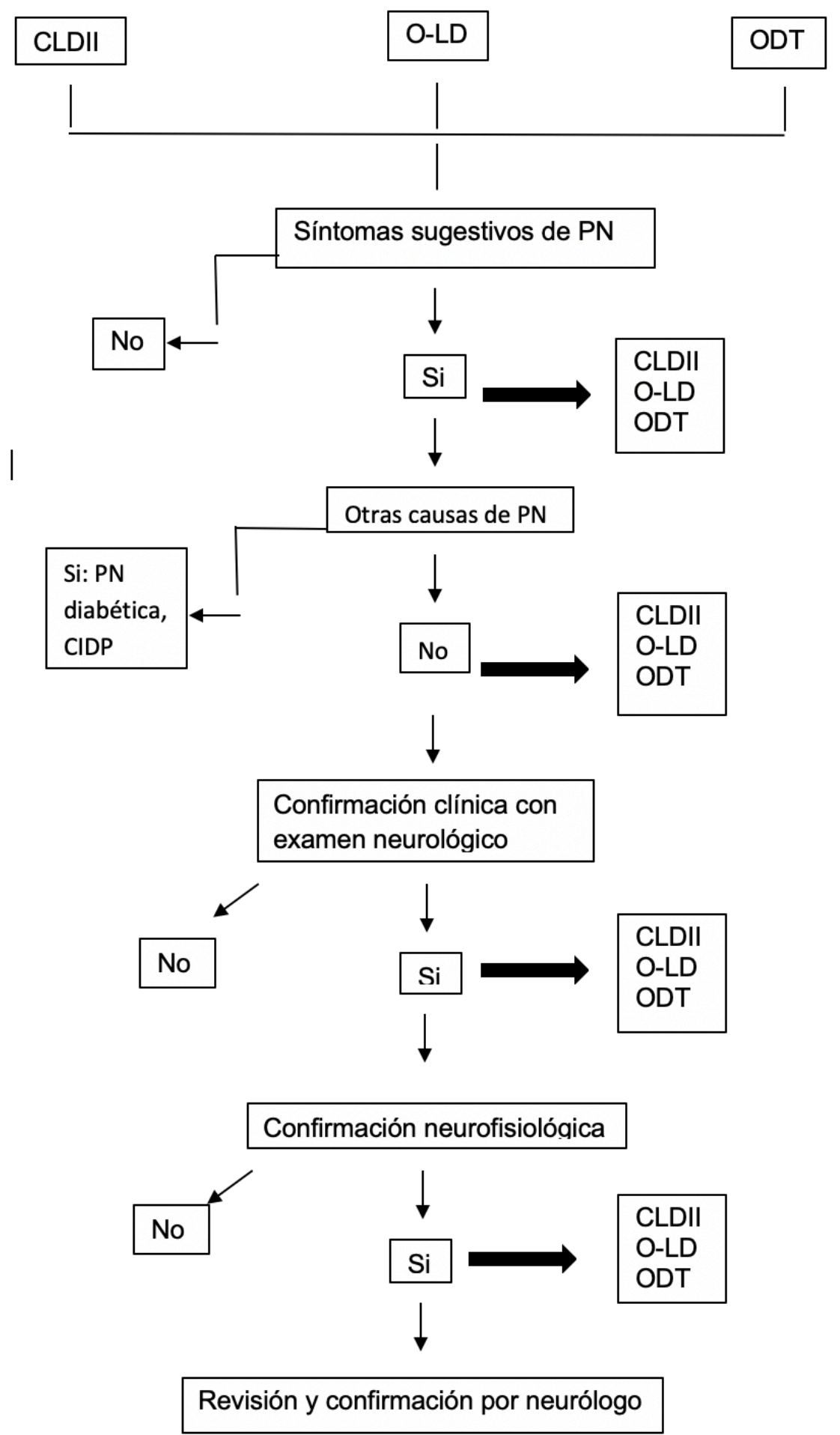

CLDII: administración continua de levodopa por infusión intestinal, O-LD: levodopa oral, ODT: otra terapia dopaminérgica, PN: neuropatía periférica, PDIC: enfermedad desmielinizante inflamatoria crónica.

Figura 2. Flujograma diagnóstico para determinar la presencia de neuropatía periférica por uso de levodopa (19). 
condición y el diagnóstico se puede establecer como neuropatía probable con controles electrofisiológicos periódicos (2).

Recientemente se demostró que la microscopia con focal corneal apoya al diagnóstico no invasivo de neuropatía de fibras pequeñas en la EP, a diferencia de la biopsia de piel que aunque más preciso, es un método más cruento (17). Debemos de tener en cuenta que un grupo muy significativo de pacientes con neuropatía en la EP no están asociados al uso de levodopa por lo tanto la ausencia de la toma de este fármaco no debería hacer que descartemos la posibilidad de estar frente a esta entidad clínica (1).

En caso de sospecha de polineuropatía aguda o subaguda, el diagnóstico se basa en las manifestaciones clínicas, los pocos casos registrados se han visto generalmente en pacientes con enfermedad de Parkinson avanzada, con neuropatía axonal sensitivomotora $(11,15)$.

\section{Manejo de la neuropatía en la enfermedad de Parkinson}

Como la etiología es aun parcialmente conocida, se sugiere un enfoque empírico, para esto se recomienda un manejo administrando suplemento parenteral de vitamina B12 1 en ampollas (5000 mcg) por día durante 7 días al comienzo del tratamiento, luego seguir con 1 ampolla por mes como dosis de mantenimiento, así mismo se recomienda suplemento oral de vitamina B1. También se recomienda el uso de folatos vía oral, en caso de demostrar su deficiencia, en ciclos con una dosis de $5 \mathrm{mg}$ por día (11).

En cuanto al uso de ICOMT se puede considerar entre las posibles estrategias de prevención de neuropatía relacionada con levodopa, pero debe ser precedido de una valoración riesgo - beneficio por su capacidad para producir discinesias. En los casos de polineuropatía crónica no se recomienda suspender la levodopa ya sea la vía oral o la infusión del gel intestinal (7).

En los casos de polineuropatías agudas o subagudas tipo síndrome de Guillain-Barré algunos autores recomiendan suspender la levodopa ya sea la vía oral o la infusión en gel intestinal de levodopa/ carbidopa, y considerar el uso de plasmaféresis o de inmunoglobulinas, así como el uso de suplemento de vitamina B12 y folatos (11).

\section{CONCLUSIONES}

El estudio y manejo terapeutico del compromiso del nervio periférico en pacientes con EP es relativamente reciente y continúa siendo muy controvertido. La fisiopatología subyacente es tema de grandes debates, su etiología y pronóstico, son temas poco dilucidados, entenderlos nos podría llevar a comprender la ansiada fisiopatología de la enfermedad de Parkinson revelándonos gran parte de los misterios que aún enmarca. La Levodopa vía oral o en infusión en gel de levodopa/carbidopa están asociadas a polineuropatía por disminuir las vitaminas B6, B12, folatos, y/o por aumentar la homocisteína y alterar la absorción y metabolismo de levodopa.

Podría existir neuropatía en los diferentes estadios de la enfermedad de Parkinson, por lo tanto, se deben monitorizar los síntomas asociados a la misma, dolor, parestesias distales y simétricas, alteración de percepción de la temperatura, reflejos, ataxia, desde el inicio del examen clínico del paciente.

El monitoreo electrofisiológico debe realizarse ante la sospecha clínica y en relación a los factores asociados ya descritos, su normalidad no descarta la presencia de polineuropatía, y también apoya en el seguimiento del paciente.

El manejo actual es suplemento de vitaminas B12, folatos, en caso encontremos déficit del mismo, en algunos casos retirar la levodopa y valorar uso de plasmaféresis o inmunoglobulinas sólo en casos de polineuropatía aguda y subaguda más no en la crónica, y el uso de ICOM está aún en estudio.

\section{Correspondencia}

Marcela Alvarado

Correo electrónico: alvaradomarcelam@hotmail.com

\section{REFERENCIAS BIBLIOGRAFICAS}

1. Ceravolo R, Cossu G, Bandettini-di-Poggio M, et al. Neuropathy and levodopa in parkinson's disease: Evidence from a multicenter study. Movement Disorders. 2013;28(10):1391-1397.

2. Podgorny P, Toth C. Parkinson's Disease and Peripheral Neuropathy. InTech. 2014; 4:101-118.

3. Conradt C, Guo D, Miclea A, et al. Increased Prevalence of Polyneuropathy in Parkinson's Disease Patients: An Observational Study. Journal of Parkinson's Disease. 2018;18:141-144.

4. Yardimci N, Cemeroglu O, Ozturk E, et al. Median 
and Ulnar Neuropathy Assessment in Parkinson's Disease regarding Symptom Severity and Asymmetry. Parkinsons Dis. 2016;2016:1-7.

5. Doppler K, Ebert S, Üçeyler N, et al. Cutaneous neuropathy in Parkinson's disease: a window into brain pathology. Acta Neuropathol. 2014;128:99109.

6. Cossu G, Melis M. The peripheral nerve involvement in Parkinson Disease: A multifaceted phenomenon. Parkinsonism and Related Disorders. 2016;1:1-4.

7. Merola A, Romagnolo A, Zibetti M, Bernardini A, Cocito D, Lopiano L. Peripheral neuropathy associated with levodopa-carbidopa intestinal infusion: a long-term prospective assessment. European Journal of Neurology. 2016; 23:501-509.

8. Kimber T, Antonini A. Peripheral neuropathy and levodopa therapy in Parkinson disease: novel insights. Eur J Neurol. 2016;23(3):435-6.

9. Mancini F, Comi C, Oggioni G, et al. Prevalence and features of peripheral neuropathy in Parkinson's disease patients under different therapeutic regimens. Parkinsonism Relat Disord. 2014;20(1):27-31.

10. Yusuf A, Martey J. Neuropathy in Parkinson disease. Neurology. 2011; 77:1947-1950.

11. Müller T, van-Laar T, Cornblath D, et al. Peripheral neuropathy in Parkinson's disease: Levodopa exposure and implications for duodenal delivery. Parkinsonism and Related Disorders. 2013; 19:501-507.

12. Comi C, Magistrelli L, Oggioni G, et al. Peripheral nervous system involvement in Parkinson's disease: Evidence and controversies. Parkinsonism and Related Disorders. 2014;1:1-5.
13. Kouroupi G, Taoufik E, Vlachos I, et al. Defective synaptic connectivity and axonal neuropathology in a human iPSC-based model of familial Parkinson's disease. Proc Natl Acad Sci USA. 2017;114(18):36793688.

14. Vital A, Lepreux S, Vital C. Peripheral neuropathy and parkinsonism: a large clinical and pathogenic spectrum. J Peripher Nerv Syst. 2014;19(4):333-42.

15. Szadejko K, Dziewiatowski K, Szabat K, et al. Polyneuropathy in levodopa-treated Parkinson's patients. J Neurol Sci. 2016 Dec 15;371:36-41.

16. Jugel C, Ehlen F, Taskin B, Marzinzik F, Müller T, Klostermann F. Neuropathy in Parkinson's Disease Patients with Intestinal Levodopa Infusion versus Oral Drugs. PLoS ONE. 2013; 8:1-6.

17. Zis P, Grünewald R, Chaudhuri R, Hadjivassiliou M. Peripheral neuropathy in idiopathic Parkinson's disease: A systematic review. J Neurol Sci. 2017;378:204-209.

18. Shahrizaila N, Mahamad U, Yap A, et al. Is chronic levodopa therapy associated with distal symmetric polyneuropathy in Parkinson's disease?. Parkinsonism and Related Disorders. 2013; 19:391-393.

19. Santos-García D, Fuente-Fernández R, Valldeoriola F, et al. Polyneuropathy while on duodenal levodopa infusion in Parkinson's disease patients: we must be alert. J Neurol. 2012; 259:1668-1672.

Recibido: 27/01/2020

Aceptado: 13/12/2020 